\title{
Design of two-dimensional FIR digital filters by McClellan transformation and least-squares contour mapping
}

\author{
Soo-Chang Pei ${ }^{\mathrm{a}, *}$, Jong-Jy Shyu ${ }^{\mathrm{b}}$ \\ ${ }^{a}$ Department of Electrical Engineering, National Taiwan University, Taipei, Taiwan, Republic of China \\ ${ }^{b}$ Department of Computer Science and Engineering, Tatung Institute of Technology, Taipei, Taiwan, Republic of China
}

Received 13 September 1993; revised 11 March 1994 and 22 December 1994

\begin{abstract}
A new method for searching the coefficients of the McClellan transformation is proposed for designing 2-D FIR digital filters in this paper. It can design arbitrary shape transformation contours to map from 1-D prototypes to 2-D FIR filters very effectively. Certain conditions are composed such that the scaling problem of the transformation can be avoided. Also, a new generalized McClellan transformation is presented, so it can be applied to design 2-D filters with complex coefficients. Several examples including fan filters with arbitrary inclination, elliptically symmetric filters with arbitrary orientation, and full-plane complex filters are presented, which demonstrates the effectiveness of this method.
\end{abstract}

\section{Zusammenfassung}

Für den Entwurf von 2D-FIR-Filtern wird eine neue Methode zur Suche nach den Koeffizienten der McClellan Transformation vorgeschlagen. Damit können Transformationskonturen beliebiger Gestalt zum Übergang von IDPrototypen auf 2D-FIR-Filter entworfen werden. Es werden bestimmte Bedingungen eingebracht, so daß das Skalierungsproblem der Transformation vermieden werden kann. Weiterhin wird eine verallgemeinerte McClellan Transformation vorgestellt, die auf den Entwurf von 2D-Filtern mit komplexen Koeffizienten angewendet werden kann. Mehrere Beispiele einschließlich FAN-Filter mit beliebiger Inklination, elliptische symmetrische Filter mit beliebiger Orientierung sowie Full-Plane komplexe Filter werden wiedergegeben, wodurch die Effizienz der Methode verdeutlicht wird.

\section{Résumé}

Nous proposons dans cet article une méthode nouvelle de recherche des coefficients de la transformation de McClellan pour la conception de filters numériques FIR 2-D. Elle permet la conception de contours de transformation de forme arbitraire afin d'étendre des prototypes 1-D à des filters 2-D de manière très effective. Certaines conditions sont composées de telle sorte que le problème d'échelle de la transformation peut être évité. De plus, une transformation de McClellan généralisée nouvelle est présentée, celle-ci pouvant être appliquée à la conception de filtres 2-D à coefficients complexes. Plusieurs exemples incluant des filtres en éventail à inclinaison arbitraire, des filtres elliptiques symétriques à orientation arbitraire, et des filtres complexes sont présentés, ce qui met en évidence l'efficience de cette méthode.

*Corresponding author. 
Keywords: Analytical least-squares approach; McClellan transformation; 2-D FIR digital filter; Fan-type filter; 2-D complex filter

\section{Introduction}

McClellan has proposed a powerful technique for the design of 2-D FIR digital filters by means of 1-D to 2-D frequency transformation [4]. It consists of mapping 1-D prototype filters into 2-D filters by a change of variables. McClellan's transformation procedure breaks the 2-D filter design problem up into two smaller problems, namely, designing the transformation mapping contours and designing the 1-D prototype filters. The main advantage of this technique is that it is very fast, because the computing time is devoted almost entirely to the 1-D design. Furthermore, these 2-D filters have a very efficient structure for hardware implementation $[5,7]$.

The original McClellan transformation involves the 1-D to $2-\mathrm{D}$ operation

$$
\begin{aligned}
\cos (\omega)= & s(0,0)+s(1,0) \cos \left(\omega_{1}\right)+s(0,1) \cos \left(\omega_{2}\right) \\
& +s(1,1) \cos \left(\omega_{1}\right) \cos \left(\omega_{2}\right) .
\end{aligned}
$$

The transform in (1) was used for the design of 2-D linear phase FIR filters, and is extended lately to

$$
\begin{aligned}
\cos (\omega)= & \sum_{n_{1}=}^{I} \sum_{n_{2}=0}^{J} s\left(n_{1}, n_{2}\right) \cos \left(n_{1} \omega_{1}\right) \cos \left(n_{2} \omega_{2}\right) \\
& +\sum_{n_{1}=1}^{K} \sum_{n_{2}=1}^{L} t\left(n_{1}, n_{2}\right) \sin \left(n_{1} \omega_{1}\right) \sin \left(n_{2} \omega_{2}\right)
\end{aligned}
$$

by Mersereau et al. [7] and Nguyen et al. [9] for designing centro-symmetric 2-D filters.

In general, the coefficients of the McClellan transformation are computed using optimization techniques $[7,12]$. These techniques require a large computational effort. Recently, an eigen-approximation technique is proposed for calculation of the McClellan transformation coefficients [10], but the results are not optimal in truely least squares sense. In this paper, a new method based on the analytical least-squares method [1] is proposed for fast calculation of the McClellan transformation coefficients.
The method is optimal in truely least-squares sense and it is shown that this technique gives better results than those reported by the recent literature [10]. Also the technique can put certain constraints on the transform coefficients such that the scaling of the transformation $[7,8]$ can be avoided. Furthermore, we propose the general transformation for designing 2-D filters with complex coefficients.

This paper is organized as follows. Section 2 describes the design of 2-D quadrantally fan filters with arbitrary inclination, in which we shall present the application of the analytical least-squares method for determining the coefficients of the McClellan transformation. The proposed technique can also be used to design elliptically symmetric filters with arbitrary orientation. As to the design of complex filters, it is shown in Section 3 in which a new transformation is introduced. Finally, the conclusions are given in Section 4.

\section{Design of 2-D FIR fan filters with arbitrary inclination}

The fan-type filters are widely used to process geoseismic data. It has the ability to pass seismic events whose apparant velocities on the earth's surface fall within a wedge shaped region in the frequency wave number plane [11]. In this section, the transformation of (1) is used. It is desired to give the following constraints: (i) the 1-D frequency origin, $\omega=0$, maps to the $(0, \pi)$ point of the $2-D$ frequency plane, and (ii) the $\pi$ point of the $1-D$ frequency, $\omega=\pi$, maps to the $(\pi, 0)$ point of the 2-D frequency plane, which result in

$s(0,0)=s(1,1)$

and

$s(1,0)=1+s(0,1)$. 
Hence, the transformation becomes

$$
\begin{aligned}
\cos (\omega)= & s(1,1)\left(1+\cos \left(\omega_{1}\right) \cos \left(\omega_{2}\right)\right) \\
& +s(0,1)\left(\cos \left(\omega_{1}\right)+\cos \left(\omega_{2}\right)\right)+\cos \left(\omega_{1}\right)
\end{aligned}
$$

Now, the problem we face is to approximate $\cos \left(\omega_{\mathbf{c}}\right)-\cos \left(\omega_{1}\right)$ by $s(1,1)\left(1+\cos \left(\omega_{1}\right) \cos \left(\omega_{2}\right)\right)$ $+s(0,1)\left(\cos \left(\omega_{1}\right)+\cos \left(\omega_{2}\right)\right)$ along the cutoff line

$\omega_{2}=\tan (\theta) \omega_{1}=\rho \omega_{1}$

by selecting appropriate values of $s(1,1)$ and $s(0,1)$, where $\omega_{\mathrm{c}}$ is the cutoff frequency of $1-\mathrm{D}$ prototype filter.

The object error function which is used in [1] is defined by

$E=\sum_{k=0}^{M}\left[d\left(\omega_{1 k}, \omega_{2 k}\right)-p\left(\omega_{1 k}, \omega_{2 k}\right)\right]^{2}$,

where $\left(\omega_{1 k}, \omega_{2 k}\right), 0 \leqslant k \leqslant M$, are the grid points selected along the desired contour, $d\left(\omega_{1 k}, \omega_{2 k}\right)$ is the desired response given by

$d\left(\omega_{1 k}, \omega_{2 k}\right)=\cos \left(\omega_{\mathrm{c}}\right)-\cos \left(\omega_{1 k}\right), \quad 0 \leqslant k \leqslant M$,

and

$$
\begin{aligned}
& p\left(\omega_{1 k}, \omega_{2 k}\right) \\
& \quad=\left[\begin{array}{ll}
s(1,1) & s(0,1)
\end{array}\right]\left[\begin{array}{c}
1+\cos \left(\omega_{1 k}\right) \cos \left(\omega_{2 k}\right) \\
\cos \left(\omega_{1 k}\right)+\cos \left(\omega_{2 k}\right)
\end{array}\right] \\
& =\boldsymbol{S}^{\mathrm{T}} \boldsymbol{C}\left(\omega_{1 k}, \omega_{2 k}\right), \quad 0 \leqslant k \leqslant M .
\end{aligned}
$$

In matrix notation,

$$
\begin{aligned}
E & =\operatorname{tr}\left[(\boldsymbol{D}-\boldsymbol{P})^{\mathrm{T}}(\boldsymbol{D}-\boldsymbol{P})\right] \\
& =\operatorname{tr}\left[\boldsymbol{D}^{\mathrm{T}} \boldsymbol{D}-\boldsymbol{D}^{\mathrm{T}} \boldsymbol{P}-\boldsymbol{P}^{\mathrm{T}} \boldsymbol{D}+\boldsymbol{P}^{\mathrm{T}} \boldsymbol{P}\right]
\end{aligned}
$$

where

$$
\boldsymbol{D}=\left[\begin{array}{c}
d\left(\omega_{10}, \omega_{20}\right) \\
d\left(\omega_{11}, \omega_{21}\right) \\
\vdots \\
d\left(\omega_{1 M}, \omega_{2 M}\right)
\end{array}\right]
$$

and

$$
\boldsymbol{P}=\left[\begin{array}{c}
p\left(\omega_{10}, \omega_{20}\right) \\
p\left(\omega_{11}, \omega_{21}\right) \\
\vdots \\
p\left(\omega_{1 M}, \omega_{2 M}\right)
\end{array}\right]=\boldsymbol{C S}
$$

in which

$$
\boldsymbol{C}=\left[\begin{array}{c}
C^{\mathrm{T}}\left(\omega_{10}, \omega_{20}\right) \\
C^{\mathrm{T}}\left(\omega_{11}, \omega_{21}\right) \\
\vdots \\
C^{\mathrm{T}}\left(\omega_{1 M}, \omega_{2 M}\right)
\end{array}\right] .
$$

From (10) and (12),

$$
E=\operatorname{tr}\left[\boldsymbol{D}^{\mathrm{T}} \boldsymbol{D}-\boldsymbol{D}^{\mathrm{T}} \boldsymbol{C S}-(\boldsymbol{C S})^{\mathrm{T}} \boldsymbol{D}+(\boldsymbol{C S})^{\mathrm{T}} \boldsymbol{C S}\right] .
$$

By setting $\partial E / \partial S=0$, the optimal solution is obtained as follows:

$\boldsymbol{S}=\left(\boldsymbol{C}^{\mathrm{T}} \boldsymbol{C}\right)^{-1} \boldsymbol{C}^{\mathrm{T}} \boldsymbol{D}$.

By changing the cutoff frequency $\omega_{\mathrm{c}}$ we can obtain several sets of transformation coefficients, but we only choose the one with global minimum error. For example, if $\theta=40^{\circ}$, the plot of the error $E$ corresponding $\omega_{\mathrm{c}}$ is given in Fig. 1(a). It is found that the global minimum error occurs when $\omega_{\mathrm{c}}=$ $(0.2877) 2 \pi$, and the transformation coefficients $s(1,1)=-0.0962076, s(0,1)=-0.5257558$, and the iso-potential contours are shown in Fig. 1(b).

For comparison, 2-D fan filters with different inclination are designed, the coefficients and the respective cutoff frequency of 1-D prototype filter are tabulated in Table 1. In Table 1, the deviation of the cutoff iso-potential with the ideal one is defined by

$$
\begin{aligned}
& D=\left\{\sum _ { n = 0 } ^ { N } \left[\cos \left(\omega_{\mathrm{c}}\right)-s(0,0)-s(1,0) \cos \left(\omega_{1}\right)\right.\right. \\
& \left.\left.\quad-s(0,1) \cos \left(\omega_{2}\right)-s(1,1) \cos \left(\omega_{1}\right) \cos \left(\omega_{2}\right)\right]^{2}\right\}^{1 / 2} \\
& \text { for } \omega_{1}=\frac{n \pi}{N}, \omega_{2}=\frac{\rho n \pi}{N},
\end{aligned}
$$

where $N$ is set to 50 in this example. It is found that the errors are smaller than those of [10]. 

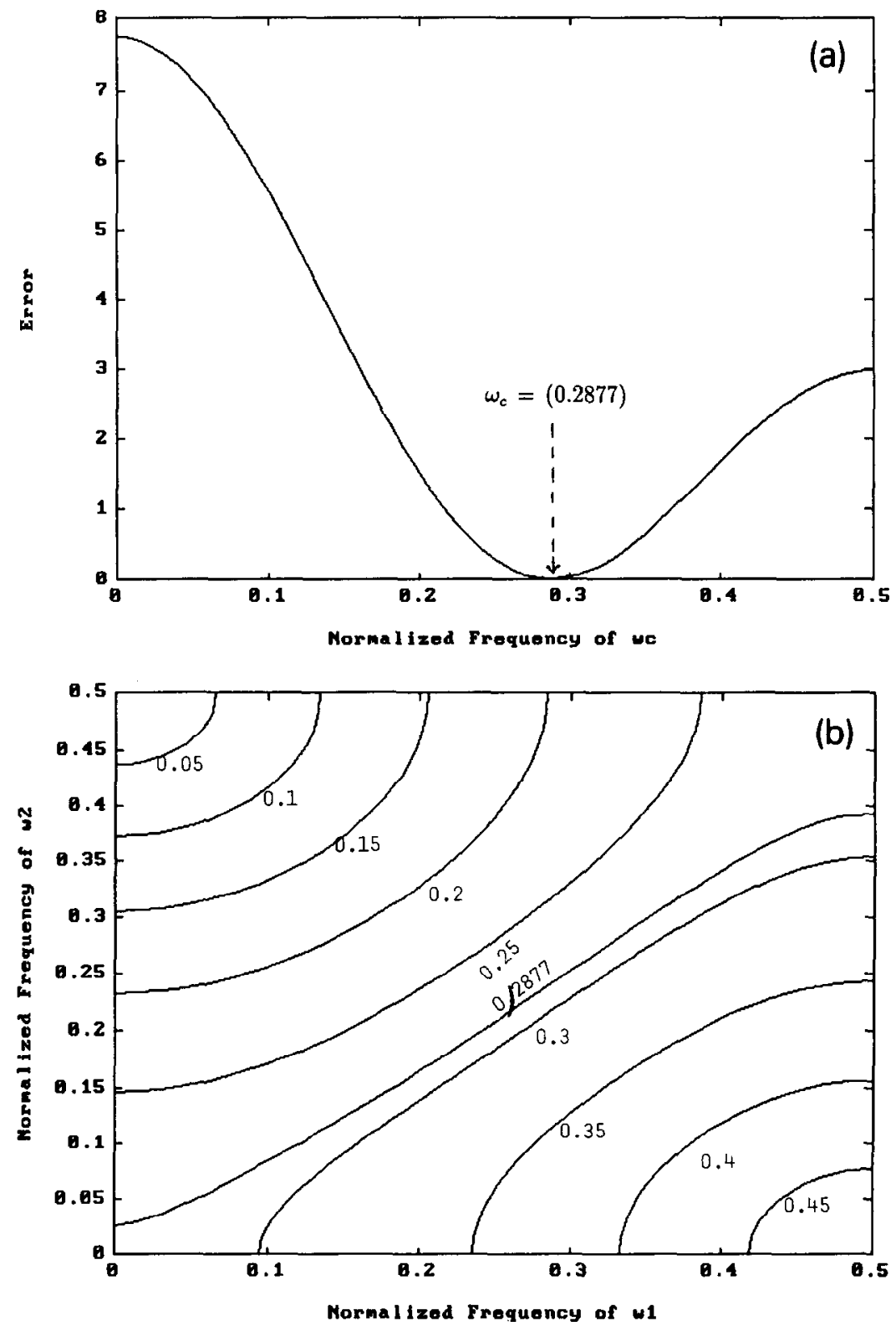

Fig. 1. Fan filter design with $\theta=40^{\circ}$, (a) error curve corresponding to $\omega_{\mathrm{c}}$, (b) iso-potential contours.

Similarly, the proposed technique can be applied for designing 2-D quadrantally elliptically symmetric filters and elliptically symmetric filters with arbitrary orientation. For example, the design of a quadrantally elliptically symmetric filter with minor axis $\omega_{a}=0.25 \pi$ and the major axis $\omega_{b}=0.5 \pi$ results in the following parameters:

$\omega_{\mathrm{c}}=0.25 \pi$,

$s(0,0)=-0.05538$,

$s(1,0)=0.7630511$,

$s(0,1)=0.05538$,

$s(1,1)=0.2369489$, 
Table 1

The values of McClellan transformation coefficients and the cutoff frequency of 1-D prototype filter for different values of $\theta$ for fan filter design

\begin{tabular}{rlllll}
\hline & & & & \multicolumn{3}{l}{ Deviation $D$} \\
\cline { 5 - 5 } 0 & $\omega_{\mathrm{c} / 2 \pi}$ & $s(1,1)$ & $s(0,1)$ & \multicolumn{3}{l}{ New method } & Algorithm of [10] \\
\hline 5 & 0.4760 & -0.3270614 & -0.6674756 & 0.0030531 & 0.0034161 \\
10 & 0.4518 & -0.3179154 & -0.6601532 & 0.0120592 & 0.0134611 \\
15 & 0.4272 & -0.3024373 & -0.6479343 & 0.026509 & 0.0294736 \\
20 & 0.4021 & -0.2808006 & -0.6305461 & 0.0453457 & 0.0502068 \\
25 & 0.3761 & -0.2513659 & -0.6089064 & 0.0666106 & 0.0733082 \\
30 & 0.3489 & -0.2131574 & -0.583326 & 0.0865212 & 0.0945202 \\
35 & 0.3199 & -0.1635669 & -0.5549054 & 0.0974535 & 0.1054766 \\
40 & 0.2877 & -0.0962076 & -0.5257558 & 0.082643 & 0.0883657 \\
45 & 0.25 & 0.0 & -0.5 & 0.0 & 0.0 \\
\hline
\end{tabular}

and the deviation error defined by

$$
\begin{aligned}
D= & \left\{\sum _ { n = 0 } ^ { 5 0 } \left[\cos \left(\omega_{c}\right)-s(0,0)-s(1,0) \cos \left(\omega_{1}\right)\right.\right. \\
& \left.\left.-s(0,1) \cos \left(\omega_{2}\right)-s(1,1) \cos \left(\omega_{1}\right) \cos \left(\omega_{2}\right)\right]^{2}\right\}^{1 / 2}
\end{aligned}
$$

for $\omega_{1}=\left[\frac{a^{2} b^{2}}{a^{2} \tan ^{2}(\theta)+b^{2}}\right]^{1 / 2}$,

$\omega_{2}=\left[\frac{a^{2} b^{2}}{a^{2} \tan ^{2}(\theta)+b^{2}}\right]^{1 / 2} \tan (\theta), \quad \theta=\frac{n \pi}{2 N}$,

is 0.0014067 which is much smaller than 0.0031194 , the deviation error of the same example in [7].

\section{General McClellan transformation for designing 2-D complex filters}

In this section, we will present a general McClellan transformation for designing 2-D complex filters (filters with complex coefficients) which can be used to process the complex-valued signals in radar image processing. Consider the $1-D$ to $2-D$ transformation with order $\left(N_{1}, N_{2}\right)$,

$$
\begin{aligned}
\cos (\omega)= & \sum_{n_{1}=0}^{N_{1}} \sum_{n_{2}=0}^{N_{2}} s\left(n_{1}, n_{2}\right) \cos \left(n_{1} \omega_{1}\right) \cos \left(n_{2} \omega_{2}\right) \\
& +\sum_{n_{1}=0}^{N_{1}} \sum_{n_{2}=1}^{N_{2}} t\left(n_{1}, n_{2}\right) \cos \left(n_{1} \omega_{1}\right) \sin \left(n_{2} \omega_{2}\right) \\
& +\sum_{n_{1}=1}^{N_{1}} \sum_{n_{2}=0}^{N_{2}} u\left(n_{1}, n_{2}\right) \sin \left(n_{1} \omega_{1}\right) \cos \left(n_{2} \omega_{2}\right) \\
& +\sum_{n_{1}=1}^{N_{1}} \sum_{n_{2}=1}^{N_{2}} v\left(n_{1}, n_{2}\right) \sin \left(n_{1} \omega_{1}\right) \sin \left(n_{2} \omega_{2}\right)
\end{aligned}
$$

For simplicity, $N_{1}=N_{2}=1$ are used and the transformation can be represented by

$$
\begin{aligned}
\cos (\omega)= & s(0,0)+s(1,0) \cos \left(\omega_{1}\right)+s(0,1) \cos \left(\omega_{2}\right) \\
& +s(1,1) \cos \left(\omega_{1}\right) \cos \left(\omega_{2}\right)+t(0,1) \sin \left(\omega_{2}\right) \\
& +t(1,1) \cos \left(\omega_{1}\right) \sin \left(\omega_{2}\right)+u(1,0) \sin \left(\omega_{1}\right) \\
& +u(1,1) \sin \left(\omega_{1}\right) \cos \left(\omega_{2}\right) \\
& +v(1,1) \sin \left(\omega_{1}\right) \sin \left(\omega_{2}\right) .
\end{aligned}
$$


(a)
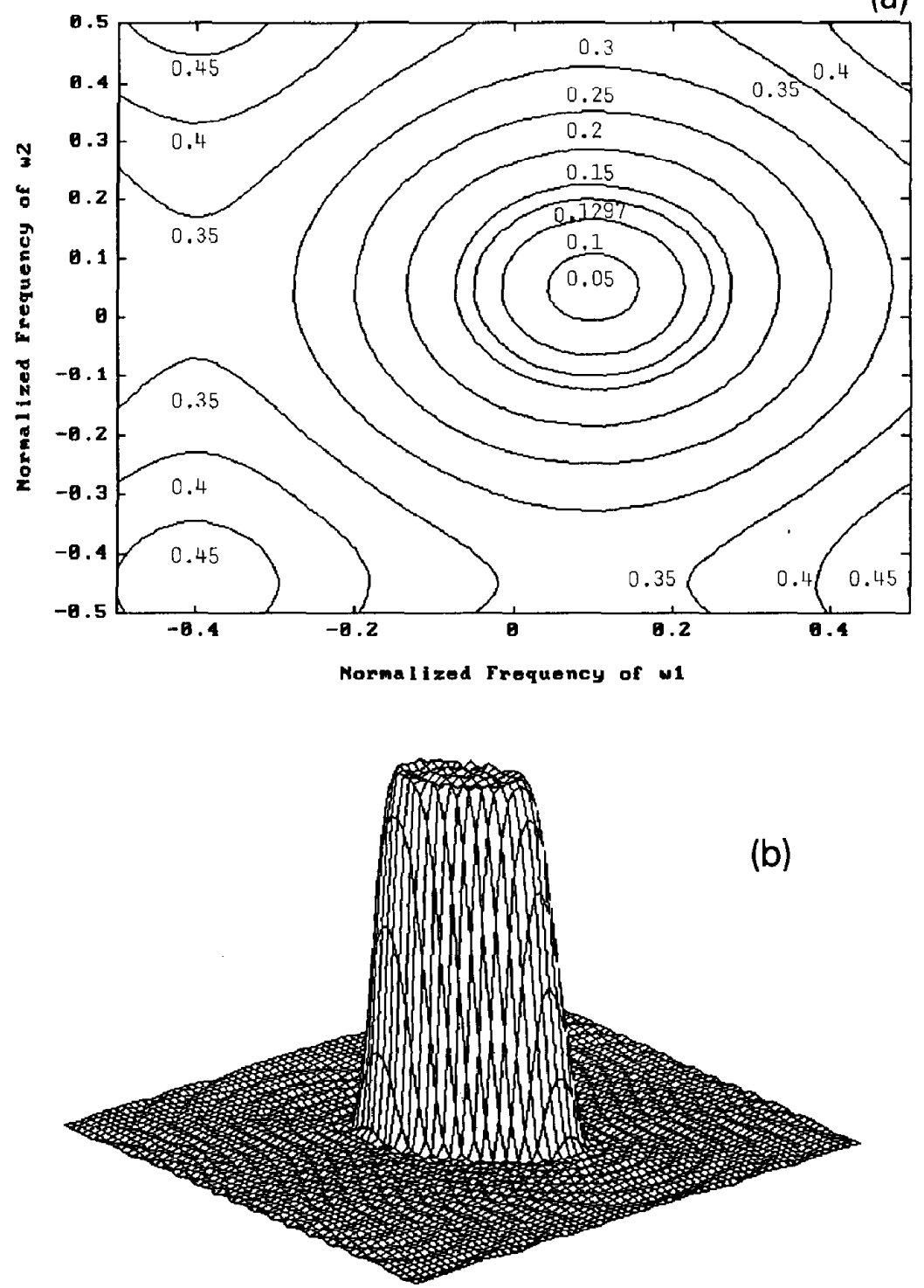

Fig. 2. A full-plane 2-D filter design, (a) iso-potential contours, (b) actual response of a $31 \times 312-\mathrm{D}$ filter.

For example, the design of a full-plane 2-D filter with the desired magnitude response

$$
\begin{aligned}
& D\left(\omega_{1}, \omega_{2}\right) \\
& = \begin{cases}1, & \sqrt{\left(\omega_{1}-\omega_{10}\right)^{2}+\left(\omega_{2}-\omega_{20}\right)^{2}} \leqslant \omega_{\mathrm{r}}, \\
0, & \sqrt{\left(\omega_{1}-\omega_{10}\right)^{2}+\left(\omega_{2}-\omega_{20}\right)^{2}} \geqslant \omega_{\mathrm{s}}\end{cases}
\end{aligned}
$$

is considered. For avoiding scaling problem, two constraints are needed: (i) $\omega=0$ maps to $\left(\omega_{10}, \omega_{20}\right)$ and (ii) $\omega=\pi$ maps to $\left(\omega_{10}+\pi, \omega_{20}+\pi\right)$, which result in

$$
\begin{aligned}
s(0,0)= & -s(1,1) \cos \left(\omega_{10}\right) \cos \left(\omega_{20}\right) \\
& -t(1,1) \cos \left(\omega_{10}\right) \sin \left(\omega_{20}\right) \\
& -u(1,1) \sin \left(\omega_{10}\right) \cos \left(\omega_{20}\right) \\
& -v(1,1) \sin \left(\omega_{10}\right) \cos \left(\omega_{20}\right)
\end{aligned}
$$


and

$$
\begin{aligned}
s(1,0)= & \frac{1}{\cos \left(\omega_{10}\right)}\left[1-s(0,1) \cos \left(\omega_{20}\right)\right. \\
& \left.-t(0,1) \sin \left(\omega_{20}\right)-u(1,0) \sin \left(\omega_{10}\right)\right]
\end{aligned}
$$

Hence, the transformation becomes

$$
\begin{aligned}
\cos (\omega)) & s(0,1)\left[\cos \left(\omega_{2}\right)-\frac{\cos \left(\omega_{20}\right)}{\cos \left(\omega_{10}\right)} \cos \left(\omega_{1}\right)\right] \\
+ & s(1,1)\left[\cos \left(\omega_{1}\right) \cos \left(\omega_{2}\right)-\cos \left(\omega_{10}\right) \cos \left(\omega_{20}\right)\right] \\
+ & t(0,1)\left[\sin \left(\omega_{2}\right)-\frac{\sin \left(\omega_{20}\right)}{\cos \left(\omega_{10}\right)} \cos \left(\omega_{1}\right)\right] \\
+ & t(1,1)\left[\cos \left(\omega_{1}\right) \sin \left(\omega_{2}\right)-\cos \left(\omega_{10}\right) \sin \left(\omega_{20}\right)\right] \\
+ & u(1,0)\left[\sin \left(\omega_{1}\right)-\frac{\sin \left(\omega_{10}\right)}{\cos \left(\omega_{10}\right)} \cos \left(\omega_{1}\right)\right] \\
+ & u(1,1)\left[\sin \left(\omega_{1}\right) \cos \left(\omega_{2}\right)-\sin \left(\omega_{10}\right) \cos \left(\omega_{20}\right)\right] \\
+ & v(1,1)\left[\sin \left(\omega_{1}\right) \sin \left(\omega_{2}\right)\right. \\
& \left.-\sin \left(\omega_{10}\right) \sin \left(\omega_{20}\right)\right]+\frac{\cos \left(\omega_{1}\right)}{\cos \left(\omega_{10}\right)} .
\end{aligned}
$$

Notice that there are only seven coefficients to be found.

If $\omega_{\mathrm{r}}=0.3 \pi$ and $\left(\omega_{10}, \omega_{20}\right)=(0.2 \pi, 0.1 \pi)$, we find that the cutoff frequency of the 1-D prototype filter is $\omega_{\mathbf{c}}=2 \pi(0.1297)$ and the transformation coefficients are

$$
\begin{aligned}
& s(0,1)=0.4755283 \\
& s(1,1)=0.2015481, \\
& t(0,1)=0.1545085, \\
& t(1,1)=0.065487, \\
& u(1,0)=0.2938923, \\
& u(1,1)=0.1464337 \\
& v(1,1)=0.0475792 .
\end{aligned}
$$

Fig. 2(a) shows the contours and Fig. 2(b) shows the actual response of a $31 \times 312$-D filter. Notice that the 1-D prototype filter with length 31 , passband $[0,(0.1297) 2 \pi]$ and stopband $[(0.18) 2 \pi, \pi]$ is used.

\section{Conclusions}

A simple method for determining the coefficients of McClellan transformation and the corresponding cutoff frequency of the $1-D$ prototype filter has been presented in this paper for designing 2-D digital filters including fan filters with arbitrary inclination and elliptically symmetric filters with arbitrary orientation. Also, the general McClellan transformation has been proposed such that it can design complex 2-D filters. Several numerical examples are given to demonstrate the usefulness and the efficiency of the present method.

\section{References}

[1] M.O. Ahmad and J.D. Wang, "An analytical least squares solution to the design problem of two-dimensional FIR filters with quadrantally symmetric and antisymmetric frequency response", IEEE Trans. Circuits Systems, Vol. 36 , July 1989, pp. 968-979.

「2ך S.N. Hazra and M.S. Reddy, "Design of circularly symmetric low-pass two-dimensional FIR digital filters using transformation", IEEE Trans. Circuits Systems, Vol. 33, October 1986, pp. 1022-1026.

[3] H.K. Kwan and C.L. Chan, "Circularly symmetric twodimensional multiplierless FIR digital filter design using an enhanced McClellan transformation", IEE Proc., Vol. 136, Part G, No. 3, June 1989, pp. $129 \cdots 134$.

[4] J.H. McClellan, "The design of two-dimensional digital filters by transformations", Proc. 7th Annual Princeton Conf. Information Sciences and Systems, 1973, pp. 247-251.

51 J.H. McClellan and D.S.K. Chan, "A 2-D FIR filter structure derived from the Chebyshev recursion", IEEE Trans. Circuits Systems, Vol. 24, July 1977, pp. $372-378$.

[6] R.M. Mersereau, "The design of two-dimensional zerophase filters using transformations", IEEE Trans. Circuits Systems, Vol. 27. February 1980, pp. 142 144.

[7] R.M. Mersereau, W.F.G. Mecklenbräuker and T.F. Quatjeri, "McClellan transformations for two-dimensional digital filters: Part I -- Design, Part II -- Implementation", IEEE Trans. Circuits Systems. Vol. 23, July 1976, pp. $405-422$.

[8] D.T. Nguyen and M.N.S. Swamy, "Formulas for parameters scaling in the McClellan transform", IEEE Trans. Circuits Systems, Vol. 33, January 1986, pp. $108 \cdots 109$. 
[9] D.T. Nguyen and M.N.S. Swamy, "Approximation design of 2-D digital filters with elliptical magnitude response of arbitrary orientation", IEEE Trans. Circuits Sysiems, Vol. 33, June 1986, pp. 597-603.

[10] S.C. Pei and J.J. Shyu, "Design of two-dimensional FIR digital filters by McClellan transformation and least squares eigen-contour mapping", IEEE Trans. Circuits Systems-II, Vol. 40, September 1993, pp. 546-555.

[11] E.Z. Psarakis, V.G. Mertzios and G.H. Alexiou, "Design of two-dimensional zero phase FIR fan filters via the
McClellan transform", IEEE Trans. Circuits Systems, Vol. 37, January 1990, pp. 10-16.

[12] P.K. Rajan and M.N.S. Swamy, "Design of circulary symmetric two-dimensional FIR filters employing transformations with variable parameters", IEEE Trans. Acoust. Speech Signal Process., Vol. 31, June 1983, pp. 637-642.

[13] M.S. Reddy and S.N. Hazra, "Design of elliptically symmetric two-dimensional FIR filters using the McClellan transformation", IEEE Trans. Circuits Systems, Vol. 34, February 1987, pp. 196-198. 